\title{
Bistability of buoyancy-driven exchange flows in vertical tubes
}

\author{
Jenny Suckale ${ }^{1, \dagger}$, Zhipeng Qin ${ }^{1}$, Davide Picchi ${ }^{2}$, Tobias Keller ${ }^{1}$ \\ and Ilenia Battiato ${ }^{2}$ \\ ${ }^{1}$ Department of Geophysics, Stanford University, Stanford, CA 94305, USA \\ ${ }^{2}$ Department of Energy Resources Engineering, Stanford University, Stanford, CA 94305, USA
}

(Received 18 January 2018; revised 3 April 2018; accepted 1 May 2018; first published online 6 July 2018)

Buoyancy-driven exchange flows are common to a variety of natural and engineering systems, ranging from persistently active volcanoes to counterflows in oceanic straits. Laboratory experiments of exchange flows have been used as surrogates to elucidate the basic features of such flows. The resulting data have been analysed and interpreted mostly through core-annular flow solutions, the most common flow configuration at finite viscosity contrasts. These models have been successful in fitting experimental data, but less effective at explaining the variability observed in natural systems. In this paper, we demonstrate that some of the variability observed in laboratory experiments and natural systems is a consequence of the inherent bistability of core-annular flow. Using a core-annular solution to the classical problem of buoyancy-driven exchange flows in vertical tubes, we identify two mathematically valid solutions at steady state: a solution with fast flow in a thin core and a solution with relatively slow flow in a thick core. The theoretical existence of two solutions, however, does not necessarily imply that the system is bistable in the sense that flow switching may occur. Through direct numerical simulations, we confirm the hypothesis that core-annular flow in vertical tubes is inherently bistable. Our simulations suggest that the bistability of core-annular flow is linked to the boundary conditions of the domain, which implies that is not possible to predict the realized flow field from the material parameters of the fluids and the tube geometry alone. Our finding that buoyancy-driven exchange flows are inherently bistable systems is consistent with previous experimental data, but is in contrast to the underlying hypothesis of previous analytical models that the solution is unique and can be identified by maximizing the flux or extremizing the dissipation in the system. Our results have important implications for data interpretation by analytical models and may also have interesting ramifications for understanding volcanic degassing.

Key words: core-annular flow, magma and lava flow, multiphase flow

† Email address for correspondence: jsuckale@stanford.edu 


\section{Introduction}

Buoyancy-driven, bidirectional flow in channels or tubes is relevant to many natural and industrial processes. Examples include lubricated pipelining that facilitates transport of viscous oil through pipelines by injecting lubricants like water (e.g. Joseph et al. 1997), the flow of cement into drilling mud in wellbores (e.g. Frigaard \& Scherzer 1998), the counterflow of currents with different densities in oceanic straits (e.g. Dalziel 1992) and magma circulation in the conduits of persistently degassing volcanoes (e.g. Stevenson \& Blake 1998).

The buoyancy-driven exchange flow between two immiscible fluids in a vertical tube is rarely if ever stable (e.g. Joseph et al. 1997). Significant effort has been devoted to identifying the various pertinent flow types, including the formation of bubbles, slugs and side-by-side flow (e.g. Joseph \& Renardy 1992; Brauner 1998). Here, we investigate the flow variability associated with core-annular flow, the most commonly observed flow regime of exchange flows in vertical pipes. The core-annular geometry is characterized by one fluid flowing in the centre of the tube (core) surrounded by a film of the other fluid wetting the tube walls (annulus).

Numerous authors have examined the linear stability of core-annular flow (e.g. Joseph et al. 1997). In the vertical configuration, Hickox (1971) studied the linear stability of Poiseuille flow in the limit of long waves, assuming the fluid viscosity in the core is less than in the annulus. Despite the instability of the flow to long waves, a high viscosity contrast and surface tension notably suppress the growth rate of interface instabilities. Preziosi, Chen \& Joseph (1989) focused on the linear stability of the flow when the less viscous fluid occupies the annulus, $\mathrm{Hu} \&$ Joseph (1989) extended their analysis to different flow arrangements and Chen, Bai \& Joseph (1990) included the effects of gravity. Chen \& Joseph (1991) considered the nonlinear stability of core-annular flows. Bai, Chen \& Joseph (1992) compared the predictions of linear stability theory with experiments and documented new flow types characterized by nearly stationary interface waves, termed bamboo and corkscrew waves, that correspond approximately to the fastest-growing wavelength, suggesting that core-annular flow reaches a metastable flow configuration despite its inherent instability.

Our study seeks to explain the variability of core-annular flow between two Newtonian fluids in a vertical tube at low Reynolds number. Our work is motivated primarily by the need to better understand degassing processes in persistently active volcanoes, the most common form of volcanism on Earth. Many persistently degassing volcanoes have been active for periods exceeding the historical record. Although erupting comparatively small amounts of lava, they continuously emit copious amounts of volatiles and thermal energy, suggesting that the majority of magma is recycled back into the plumbing system after decoupling from the gas phase near the surface (e.g. Francis, Oppenheimer \& Stevenson 1993). At steady state, the ascent of gas-rich, buoyant magma in the conduit would therefore approximately balance the simultaneous descent of gas-poor, dense magma, which would result in exchange flow in the volcanic conduit (Kazahaya, Shinohara \& Saito 1994; Stevenson \& Blake 1998; Burton, Mader \& Polacci 2007; Witham 2011).

The motivation to better understand volcanic degassing informs the parameter range we investigate in this paper. The density contrast between the two fluids is the consequence of gas bubbles exsolving from the magma. In the context of bidirectional flow, it is assumed that these gas bubbles are small compared to the radius of the conduit and remain entrained in the upwelling fluid. The resulting density difference tends to be much less variable than the viscosity ratio between the two fluids, which 
can vary by multiple orders of magnitude. For example, exsolution of 1 wt. \% water typically increases the viscosity of the silicate melt by approximately a factor of 10 (e.g. McBirney \& Murase 1984). Volatile loss also tends to be associated with crystallization, which can further significantly increase the effective viscosity of the degassed magma (e.g. McBirney \& Murase 1984). Our main emphasis is hence on bidirectional flow with high viscosity contrast in vertical, or close to vertical, pipes, building on work by Joseph, Renardy \& Renardy (1984), but at very low Reynolds number.

In the volcanic context, the upwelling magma in the core is typically less viscous than the downwelling magma in the annulus, because it is more volatile rich. This arrangement is contrary to the typical arrangement in lubricated pipelining and tends to be unstable at finite Reynolds number, as demonstrated theoretically (Joseph et al. 1984) and experimentally (Arakeri et al. 2000; Huppert \& Hallworth 2007). However, at the low Reynolds numbers and large viscosity contrasts likely representative of volcanic systems, core-annular flow with the less viscous fluid in the core becomes stable (Hickox 1971; Joseph et al. 1984; Stevenson \& Blake 1998).

Another important difference between bidirectional flow in volcanic systems and that in lubricated pipelining is the miscibility of the two fluids involved. Despite their different properties, the upwelling and downwelling magmas in volcanic conduits are miscible. The diffusivities of the magmas, however, are probably sufficiently low to mimic immiscible flows. Experimental studies have shown that miscible bidirectional flow at low diffusivity exhibits similar flow regimes as immiscible flow (Petitjeans \& Maxworthy 1996; Scoffoni, Lajeunesse \& Homsy 2001), including the characteristic corkscrew waves at the fluid interface observed in the immiscible context (e.g. Bai et al. 1992; Hu \& Patankar 1995; Renardy 1997). Linear stability analysis, however, has suggested that even a small degree of mixing at the interface can stabilize the flow over a wide range of conditions (Vanaparthy, Meiburg \& Wilhelm 2003; Meiburg et al. 2004).

Our work builds on laboratory experiments of exchange flow in closed vertical tubes conducted by Stevenson \& Blake (1998) and Beckett et al. (2011). We focus on this specific set of experiments because the parameter range is representative of volcanic systems. We analyse the flow behaviour observed in the laboratory by using direct numerical simulations to virtually reproduce the original experiments. Our numerical model does not require closures such as drag forces, interface stresses or rise speeds (Suckale et al. 2010a; Suckale, Nave \& Hager 2010b; Qin \& Suckale 2017). Instead, these flow properties emerge self-consistently from the computation, as in analogue experiments where the flow dynamics emerges directly from the experimental set-up and the materials used. Direct numerical simulations hence enable us to quantify velocities, stresses, and other flow variables at all times and locations in the flow field and to extend experiments to scales or flow conditions not realizable in a laboratory setting.

We link our direct numerical simulations to an analytical model of laminar core-annular flow in a vertical tube at steady state, building on previous similar approaches (e.g. Russell \& Charles 1959; Kazahaya et al. 1994; Ullmann \& Brauner 2004; Huppert \& Hallworth 2007). We predict the existence of two distinct solutions at steady-state conditions: a solution with fast flow in a thin core and a solution with relatively slow flow in a thick core. The existence of two solutions suggests that exchange flow in vertical tubes is a bistable problem. We test this hypothesis through direct numerical simulations $(\$ 2.2)$ and find that the boundary conditions control which solution is realized in a laboratory experiment $(\S 3)$. This insight implies that the bidirectional flow regime in vertical tubes cannot be predicted based on 
(a)

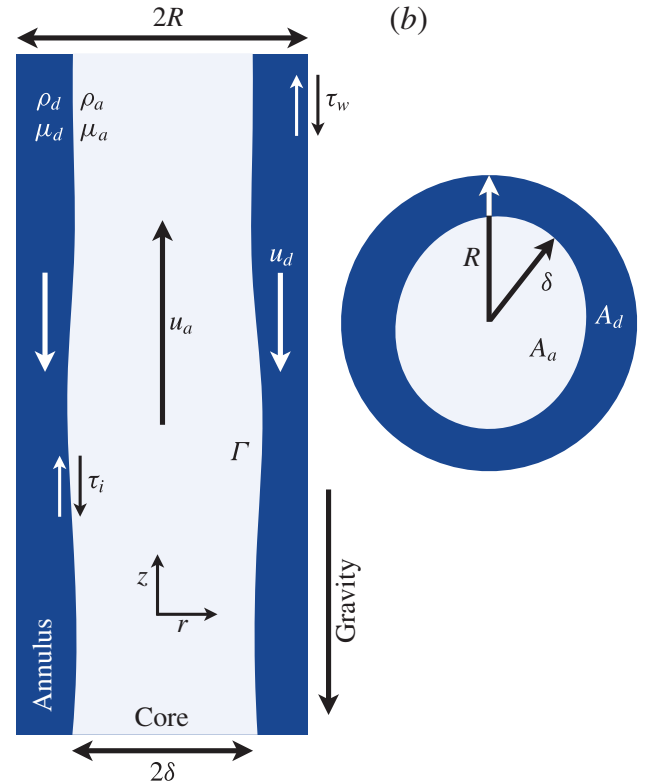

FIGURE 1. Sketch of the core-annular geometry and key variables used for a vertical $(a)$ and a horizontal $(b)$ cross-section. The interface between the two fluids is depicted as wavy to highlight the unstable nature of the flow pattern.

geometry and material parameters alone - an aspect of core-annular flow that could have important implications for understanding the variability of magma circulation in volcanic conduits and the resulting eruptive activity $(\$ 4)$.

\section{Model description}

We combine two distinct and complementary model components: an analytical model of core-annular flow at steady state, and direct numerical simulations of exchange flow in two dimensions. We focus on two-dimensional (2-D) numerical simulations to afford higher resolution of the evolving interface. Whereas the analytical model is limited to core-annular flow of immiscible fluids, our numerical approach can capture the various flow regimes that arise for both the miscible fluids employed in the laboratory experiments and the immiscible fluids assumed in the analytical model.

\subsection{Analytical model}

\subsubsection{Derivation}

At low Reynolds number, the steady-state core-annular flow of two immiscible and incompressible Newtonian fluids in a pipe of inclination $\alpha$ from the vertical direction can be described by the 1-D Stokes equations along the radial coordinate $r \in[0, R]$ (see figure 1),

$$
\begin{array}{ll}
\mu_{d} \frac{1}{r} \frac{\mathrm{d}}{\mathrm{d} r}\left(r \frac{\mathrm{d} u_{d}}{\mathrm{~d} r}\right)=\frac{\mathrm{d} p}{\mathrm{~d} z}+\rho_{d} g \cos \alpha, & r \in[\delta, R], \\
\mu_{a} \frac{1}{r} \frac{\mathrm{d}}{\mathrm{d} r}\left(r \frac{\mathrm{d} u_{a}}{\mathrm{~d} r}\right)=\frac{\mathrm{d} p}{\mathrm{~d} z}+\rho_{a} g \cos \alpha, & r \in[0, \delta],
\end{array}
$$


where the subscripts $(\cdot)_{d}$ and $(\cdot)_{a}$ denote the descending and ascending fluid properties, namely dynamic viscosity $\mu$, density $\rho$ and vertical speed $u$, respectively, $R$ is the pipe radius, $g$ the gravitational acceleration and $\delta$ represents the unknown location of the interface between the ascending and descending fluids. The pressure drop is an unknown constant to be determined. The boundary conditions are no-slip at the tube wall,

$$
u_{d}(R)=0
$$

vanishing radial stress at the symmetry line in the tube centre,

$$
\frac{\mathrm{d} u_{a}}{\mathrm{~d} r}(0)=0,
$$

and continuity of velocity and shear stress across the fluid-fluid interface,

$$
\begin{gathered}
u_{d}(\delta)-u_{a}(\delta)=0, \\
\mu_{a} \frac{\mathrm{d} u_{a}}{\mathrm{~d} r}(\delta)-\mu_{d} \frac{\mathrm{d} u_{d}}{\mathrm{~d} r}(\delta)=0 .
\end{gathered}
$$

Instead of a phase flow-rate scaling (Ullmann \& Brauner 2004), we define the nondimensional quantities

$$
\hat{r}=\frac{r}{R}, \quad \hat{\delta}=\frac{\delta}{R}, \quad \hat{u}=\frac{u}{U},
$$

where we define $U=\Delta \rho g R^{2} / \mu_{d}$, the viscous rise speed due to the density difference $\Delta \rho=\rho_{d}-\rho_{a}$. Substituting (2.6) into (2.1) and dropping all hats yields the dimensionless equations,

$$
\begin{aligned}
\frac{1}{r} \frac{\mathrm{d}}{\mathrm{d} r}\left(r \frac{\mathrm{d} u_{d}}{\partial r}\right) & =P, \quad r \in[\delta, 1], \\
\frac{1}{M} \frac{1}{r} \frac{\mathrm{d}}{\mathrm{d} r}\left(r \frac{\mathrm{d} u_{a}}{\mathrm{~d} r}\right) & =P-\cos \alpha, \quad r \in[0, \delta],
\end{aligned}
$$

where $P=\left(\mathrm{d} p / \mathrm{d} z+\rho_{d} g \cos \alpha\right) /(g \Delta \rho)$ is the non-dimensional pressure drop and $M=\mu_{d} / \mu_{a}$ is the viscosity ratio. We integrate equations (2.7), while accounting for appropriately non-dimensionalized boundary conditions, to obtain

$$
\begin{array}{ll}
u_{d}(r)=\frac{P}{4}\left(r^{2}-1\right)-\frac{\delta^{2}}{2} \cos \alpha \log r, & r \in[\delta, 1], \\
u_{a}(r)=M \frac{P-\cos \alpha}{4}\left(r^{2}-\delta^{2}\right)+u_{i}, & r \in[0, \delta],
\end{array}
$$

where $u_{i}=u_{d}(\delta)=u_{a}(\delta)$ is the vertical flow speed at the interface given by

$$
u_{i}=\frac{P}{4}\left(\delta^{2}-1\right)-\frac{\delta^{2}}{2} \cos \alpha \log \delta
$$

The ascending flux in a closed tube with incompressible fluids must exactly balance the descending flux,

$$
-\int_{\delta}^{1} 2 \pi r u_{d}(r) \mathrm{d} r=\int_{0}^{\delta} 2 \pi r u_{a}(r) \mathrm{d} r=T e,
$$


where $T e$ is the dimensionless flux or Transport number (e.g. Huppert \& Hallworth 2007). The Transport number is related, but not identical, to the Poiseuille number, Ps, defined in Stevenson \& Blake (1998),

$$
P s=\frac{u_{f} \mu_{d}}{g \Delta \rho R^{2}}=\frac{u_{f}}{U},
$$

where $u_{f}$ is the (dimensional) terminal rise speed of the ascending front (see the supplementary material available at https://doi.org/10.1017/jfm.2018.382 for details). The $P s$ number therefore represents the non-dimensional rise speed, whereas the $T e$ number captures the non-dimensional flux, and hence

$$
T e=\frac{P s}{\pi \delta^{2}} .
$$

We focus on $T e$ in our analysis, because the flux is balanced between ascending and descending fluids and does not hinge on selecting either a characteristic rise speed from the spatially variable function, $u_{a}(r)$, or a core radius, $\delta$. To fulfil the constraint of no net flux in the tube, we substitute (2.8) into (2.10) and solve for the driving force $P$, which depends only on the dimensionless parameters of the problem (i.e. $\delta$, $M, \alpha)$ :

$$
P(\delta, \alpha, M)=\delta^{2} \frac{2\left(\delta^{2}-1\right)-M \delta^{2}}{\left(\delta^{4}-1\right)-M \delta^{4}} \cos \alpha .
$$

We can now express $T e$ as a function of $P$ and $\delta$ :

$$
T e(\delta, \alpha, M)=2 \pi\left[\frac{P}{16}\left(\delta^{2}-1\right)^{2}+\frac{\delta^{2}}{8} \cos \alpha\left(\delta^{2}-1-2 \delta^{2} \log \delta\right)\right] .
$$

We note that once $P$ and $\delta$ are defined, Te is uniquely specified. The opposite, however, is not true. The quadratic and quartic terms in (2.14) suggest that a given flux, Te, could be achieved for two different $\delta$. Even though the model developed by Huppert \& Hallworth (2007) also admits multiple solutions, the authors implicitly imposed uniqueness by maximizing the flux or, alternatively, extremizing the dissipation in the system. In $\S 3$, we numerically explore the validity of this uniqueness hypothesis, characterize the differences between the two possible solutions in more detail, and investigate whether both solutions are relevant in practice.

\subsection{Numerical model}

\subsubsection{Governing equations}

Our numerical model solves for conservation of mass and momentum. We assume that both fluids are incompressible. The governing equations are hence the incompressibility condition,

$$
\nabla \cdot v=0
$$

and the variable-coefficient Navier-Stokes equation,

$$
\rho\left(\frac{\partial \boldsymbol{v}}{\partial t}+(\boldsymbol{v} \cdot \nabla) \boldsymbol{v}\right)=-\nabla p+\nabla \cdot\left[\mu\left(\nabla \boldsymbol{v}+(\nabla \boldsymbol{v})^{\mathrm{T}}\right)\right]+\rho \boldsymbol{g},
$$

where $v$ is the velocity, and $p$ the pressure. 
Density and viscosity vary in space and time to reflect the different properties of the two fluids. We consider the two contrasting scenarios of immiscible and miscible fluids. The immiscible case is useful for a direct comparison with the analytical solution, which is strictly valid only for two immiscible fluids. The miscible case represents the experimental set-up of Stevenson \& Blake (1998), which involves two miscible fluids with low diffusivity.

\subsubsection{Immiscible fluids}

In the immiscible case, a sharp interface separates the two fluids. We advect the curve, $\Gamma$, which represents this interface, with the flow field according to

$$
\frac{\partial \Gamma}{\partial t}+(\boldsymbol{v} \cdot \nabla) \Gamma=0
$$

The interface deforms in response to the hydrodynamic forces acting on it. The material properties change discontinuously at the interface:

$$
\mu(\boldsymbol{x})= \begin{cases}\mu_{a} & \text { in the ascending fluid } \\ \mu_{d} & \text { in the descending fluid }\end{cases}
$$

and

$$
\rho(\boldsymbol{x})= \begin{cases}\rho_{a} & \text { in the ascending fluid } \\ \rho_{d} & \text { in the descending fluid }\end{cases}
$$

and may entail a jump in the pressure and normal stresses at the interface:

$$
\left[\left(\begin{array}{l}
\boldsymbol{n} \\
\boldsymbol{t}_{1} \\
\boldsymbol{t}_{2}
\end{array}\right)(p \boldsymbol{I}-\boldsymbol{\tau}) \boldsymbol{n}^{\mathrm{T}}\right]=\left(\begin{array}{c}
\sigma \kappa \\
0 \\
0
\end{array}\right) .
$$

Square brackets [·] denote a jump at the fluid-fluid interface, $\boldsymbol{I}$ is the identity tensor, $\boldsymbol{\tau}=\mu\left(\boldsymbol{\nabla} \boldsymbol{v}+(\boldsymbol{\nabla v})^{\mathrm{T}}\right)$ the viscous stress tensor, $\sigma$ the surface tension, $\kappa$ the curvature of $\Gamma, \boldsymbol{n}$ the unit normal vector on $\Gamma$ pointing from the ascending towards the descending fluid and $t_{1}$ and $t_{2}$ the two unit tangential vectors on $\Gamma$. Solving the Navier-Stokes equation and introducing surface tensions implies that additional non-dimensional numbers are needed to describe the flow behaviour. We choose the Reynolds number, $R e=\Delta \rho U R / \mu_{d}$, the Bond number, $B o=\Delta \rho g R^{2} / \sigma$ and the Froude number, $F r=U^{2} /(g R)$ (see the supplementary material for a summary table). All the immiscible simulations shown in the manuscript represent the case of no surface tension, $\sigma=0$, but we discuss the effect of surface tension on interface instabilities briefly in the supplementary material.

\subsubsection{Miscible fluids}

To allow for mixing, we introduce a continuous variable, $c$, which represents the concentration of the buoyant fluid. Initially, the concentration is set to unity in the buoyant fluid and zero in the heavy fluid. The concentration evolves over time due to advection and diffusion,

$$
\frac{\partial c}{\partial t}+\boldsymbol{v} \cdot \nabla c=D \nabla^{2} c
$$




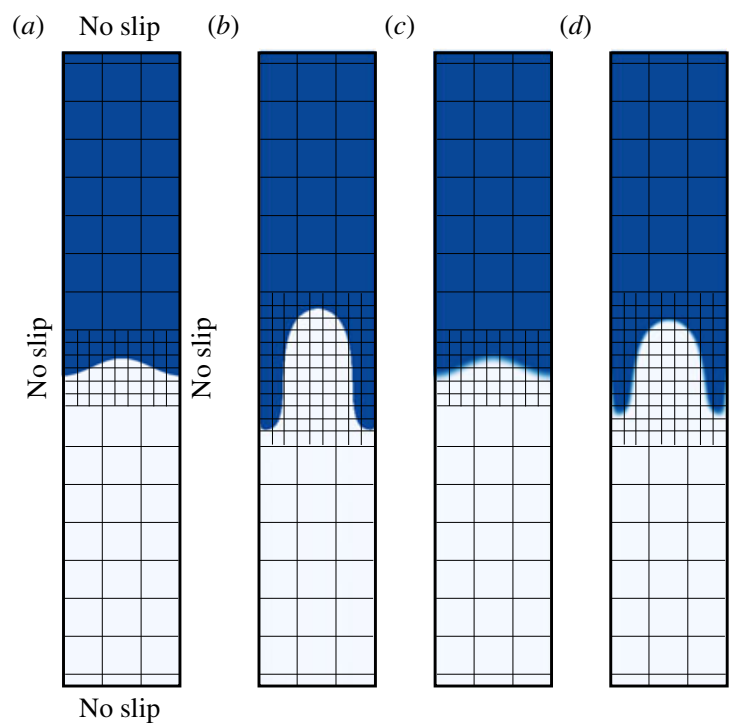

FIGURE 2. Illustration of the initial conditions, boundary conditions and adaptive grid refinement for both immiscible and miscible flow. The grid is intentionally coarse for visualization purposes. $(a, b)$ show immiscible fluids; $(c, d)$ show miscible fluids.

where $D$ is the diffusion coefficient. In their experiments, Stevenson \& Blake (1998) used miscible fluids, such as syrup, dilute syrup, glycerol and water, but did not report the fluid diffusivities. In the absence of detailed measurements, we use a constant diffusivity of $D=10^{-10} \mathrm{~m}^{2} \mathrm{~s}^{-1}$ in both fluids for all simulations, a value motivated by the diffusivity measured for corn syrup in distilled water (Ray, Bunton \& Pojman 2007). Miscibility adds another non-dimensional parameter, the Péclet number, which quantifies the relative importance of advection to diffusion $P e=R U / D$.

We assume that density and viscosity depend linearly on the concentration $c$, such that

$$
\rho=\rho_{d}+c\left(\rho_{a}-\rho_{d}\right)
$$

and

$$
\mu=\mu_{d}+c\left(\mu_{a}-\mu_{d}\right) .
$$

We note that additional complexity may arise in the vicinity of the interface if a nonlinear dependence of viscosity on concentration is assumed (see the supplementary material for details), as is the case in some prior studies (e.g. Tan \& Homsy 1986; Goyal \& Meiburg 2006).

To initialize the miscible simulations, we assume that the initial concentration field has an interface with a finite thickness of $1.5 \Delta x$, where $\Delta x$ is the coarse grid resolution (see $\S 2.2 .4$ and figure 2). Hence, no discontinuous material contrasts arise in our miscible computations.

\subsubsection{Numerical methods}

We discretize the governing equations, (2.15) and (2.16), on a Cartesian grid (see figure 2) using the numerical method derived, verified and validated in Qin \& 
Suckale (2017). For our computations, we opt for a Cartesian set-up rather than an axisymmetric formulation, because not all of the laboratory experiments we reproduce exhibit an axisymmetric symmetry and because of the importance of non-axisymmetric instabilities in core-annular flows (e.g. Hu \& Patankar 1995; Renardy 1997).

Our numerical method was developed specifically for multiphase flow problems with large viscosity contrasts at low Reynolds number (Suckale et al. 2010b, 2012). It consists of three main components. The first is a multiphase Navier-Stokes solver that handles substantial and discontinuous differences in the properties of material phases by adopting an implicit implementation of the viscous term, time-step splitting, and an approximate factorization of the sparse coefficient matrices for computational efficiency. The second component is a level-set-based interface solver that tracks the motion of a fluid-fluid interface through an iterative topology-preserving projection (Qin et al. 2015). The sharp interface solver is pertinent only if the two fluids are immiscible. In the computationally simpler case of two miscible fluids, we solve an advection-diffusion equation for concentration.

The third component is an adaptive grid refinement algorithm that increases resolution in the vicinity of the moving interface, where most of the salient physical processes originate. Accurate interface advection is highly dependent on grid resolution (e.g. Sethian 1996), particularly in flows that hinge sensitively on the growth of interface instabilities. To maximize numerical resolution at the interface, we adopt an adaptive mesh refinement strategy that tracks the interface position over time. Figure 2 shows a close up of the computational domain around the interface to schematically illustrate the grid refinement at the initial condition (figure 2a) and at a later time (figure $2 b$ ), both shown at intentionally coarse resolution for easier visualization. The refined zone extends as the interface is stretched by flow. Although the computational challenges associated with tracking a diffusive interface are less pronounced, we adopt the same grid refinement strategy for the miscible case (figure $2 c, d$ ). For more details regarding the numerical technique, including the various benchmarks performed to verify and validate the numerical method, please refer to Qin \& Suckale (2017) and this study's supplementary material.

\section{Results}

Direct numerical simulations afford the possibility to study flow behaviour beyond the scales, boundary conditions and material properties realizable in a laboratory setting. To generalize the scientific insights drawn from the laboratory work by Stevenson \& Blake (1998), we start by reproducing the original experiments, proceed to a detailed analysis of the observed flow behaviour and then generalize the experiments by allowing for flow in and out of the tubes.

\subsection{Virtual reproductions of analogue experiments}

Stevenson \& Blake (1998) initiated their experiments by inverting closed tubes filled with the denser, more viscous fluid in the lower half, and the buoyant, less viscous fluid in the upper half. We start our simulations after the inversion step, such that the two fluids are unstably stratified with a slight cosine perturbation along their interface (see figure 2). We have verified that an initial condition of different symmetry yields qualitatively equivalent model outcomes, as shown in the supplementary material. To represent the rigid glass walls of the experimental test tubes, we set all four side boundaries to no slip $(\boldsymbol{v}=0)$. All simulations are computed on a $40 \times 4000$ grid with a fourfold refinement at the interface. At this resolution, the flow dynamics is 


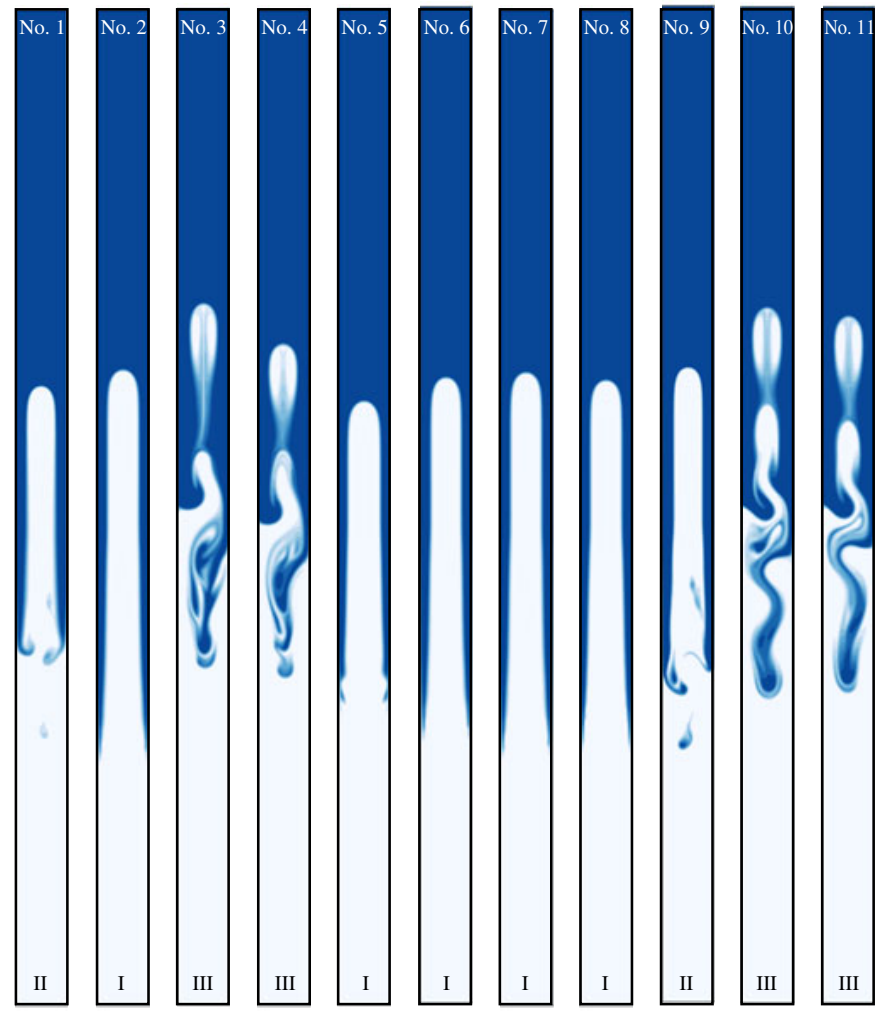

FIGURE 3. Numerical reproduction of all 11 experiments performed by Stevenson \& Blake (1998) using two miscible fluids. The dense and the buoyant fluids are shown in dark and light blue, respectively. The aspect ratio of all laboratory tubes was $1: 100$ despite different physical lengths and widths. Here and below, only the central part of the numerical domain is shown for better visibility. All simulations are shown at $t=200 \times t_{0}$, where $t_{0}=R / U$ is the non-dimensional time.

well resolved, as demonstrated by a convergence test included in the supplementary material.

We numerically reproduce all 11 analogue experiments performed by Stevenson \& Blake (1998), based on the detailed material properties they reported (see table 1). All computations are performed for two miscible fluids with low diffusivity (i.e. $D=$ $10^{-10} \mathrm{~m}^{2} \mathrm{~s}^{-1}$ ). Figure 3 shows the fully developed flows at $t=200 \times t_{0}$, where $t_{0}=$ $R / U$ is the dimensionless, viscous time scale.

We generally observe the same behaviour as reported by Stevenson \& Blake (1998), which they classified into three different overturn styles. In figure 4, we illustrate these three overturn styles in the reproduced experiments No. 8, No. 9 and No. 10. At high viscosity ratios $(M>300)$, the flow configuration is characterized by stable core-annular flow (flow regime I; figure 4a1-3). With decreasing viscosity contrast, interface waves become more pronounced. However, at intermediate viscosity ratios $(10<M<300)$, the amplitude of interface waves remains small enough to avoid wave bridging and disintegration of the core (flow regime II; figure 4b1-3). Rather, the descending fluid intermittently rips off the tube wall, while the ascending fluid forms a coherent core in the centre of the tube. Once the viscosity contrast becomes 


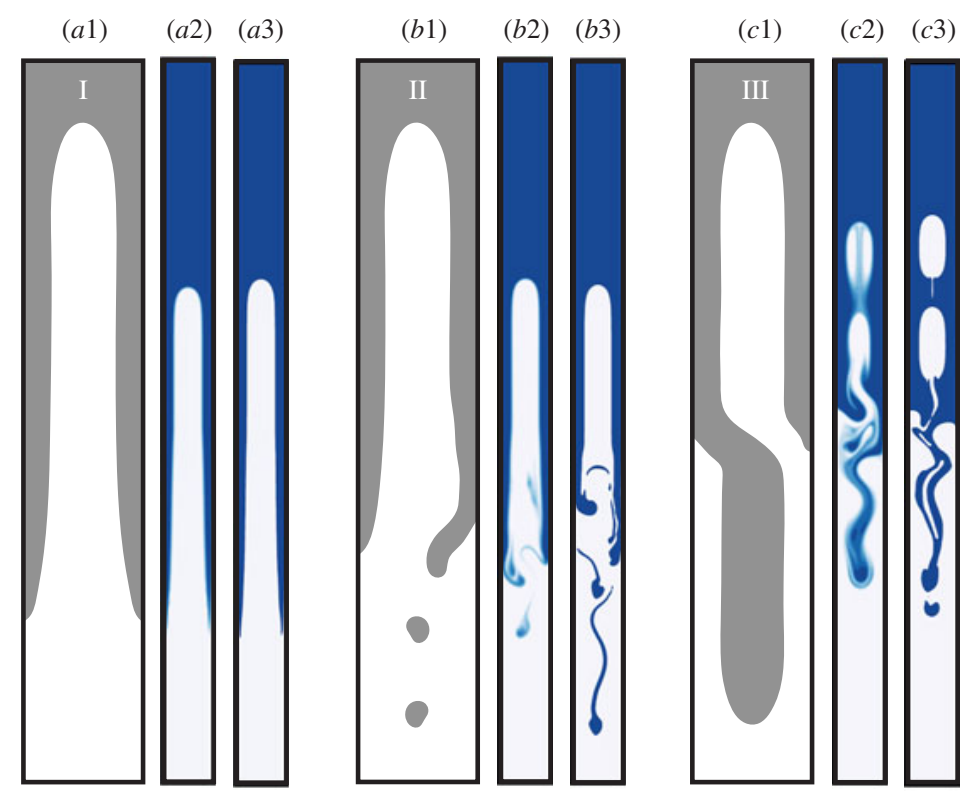

FIGURE 4. Direct numerical simulations of the three primary flow regimes (I, II, III) observed in bidirectional tube flow for different viscosity contrasts. Miscible $(a 2, b 2, c 2)$ and immiscible $(a 3, b 3, c 3)$ flows shown in comparison to schematics $(a 1, b 1, b 2)$ reproduced from Stevenson \& Blake (1998) (see also online supplementary movies 1-3 showing numerical simulations of experiments from Stevenson \& Blake). Simulation snapshots are shown at $t=200 \times t_{0}$.

small $(M<10)$, wave bridging occurs, and as a consequence, both fluids sink or rise as separate batches in the centre of the tube (flow regime III; figure $4 c 1-3$ ). We note that all three flow regimes exhibit interface waves. In agreement with the theory outlined by Hickox (1971), the amplitude and dynamic significance of these interface waves decrease as the viscosity contrast increases.

Figure 4 shows the results of three miscible simulations (figure 4a2,b2,c2) in comparison to three immiscible simulations with identical fluid densities and viscosities (figure $4 a 3, b 3, c 3$ ). The three flow regimes appear qualitatively similar for both miscible and immiscible fluids. Evidently, this conclusion holds true only for sufficiently small diffusivities, in our case for $D \leqslant 10^{-8} \mathrm{~m} \mathrm{~s}^{-2}$ or $P e>10^{2}$ (see the supplementary material for more details), which is consistent with previous estimates (Petitjeans \& Maxworthy 1996; Scoffoni et al. 2001). Figure 4 also shows that miscible flows tends to have less variability along the interface, which confirms that even a small degree of miscibility notably dampens the growth rate of interface instabilities (e.g. Meiburg et al. 2004).

The availability of an analytical solution, at least in the limit of steady core-annular flow, provides a further opportunity to validate our numerical method beyond the benchmarks presented in Qin \& Suckale (2017). More importantly, it allows us to evaluate the conditions under which miscible flow with low diffusivity behaves approximately as immiscible. In figure 5, we compare the virtual reproduction of experiment No. 8 (figure $5 a$ ) for miscible fluids with its immiscible equivalent (figure $5 b$ ) and with the analytical solution calculated for the same flow parameters. To compare the numerical and analytical results, we take horizontal profiles of 
瓜

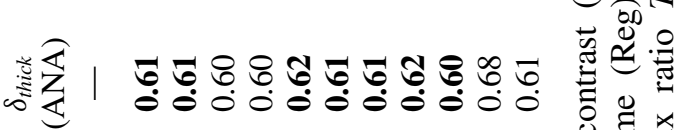

采

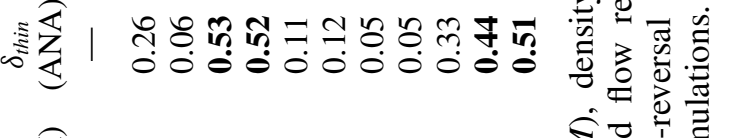

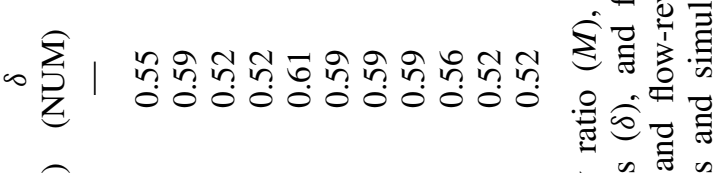

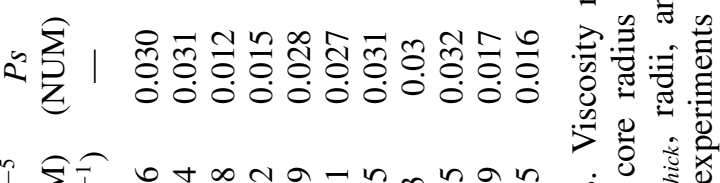

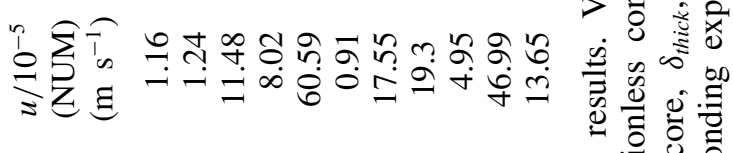

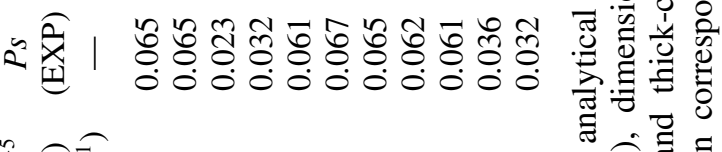

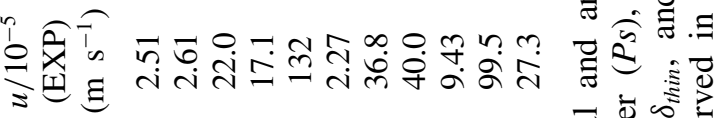

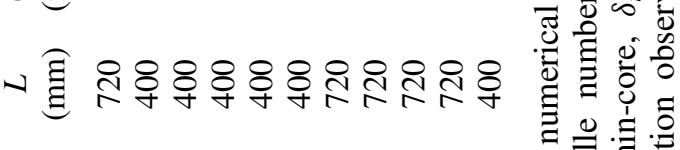

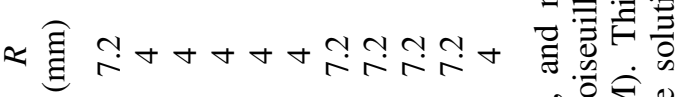

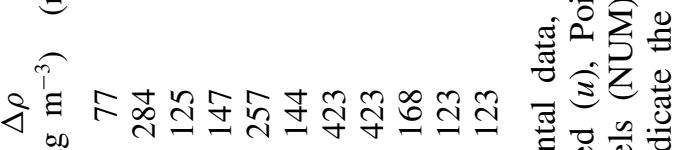

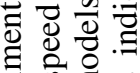

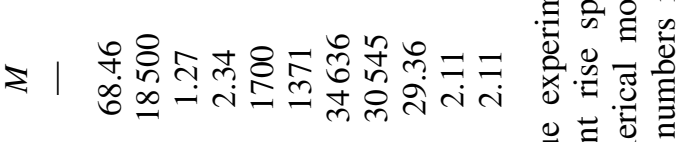

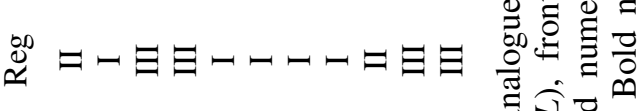

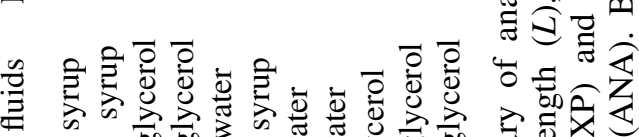

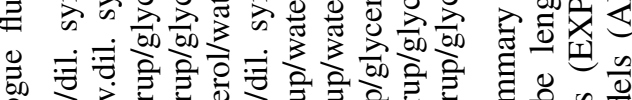

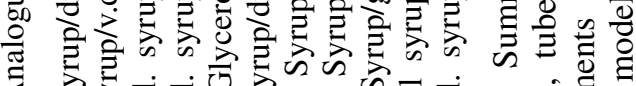

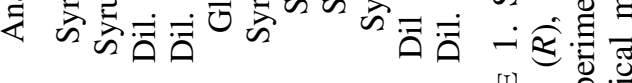

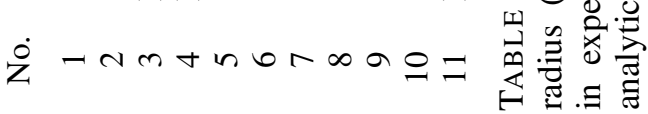



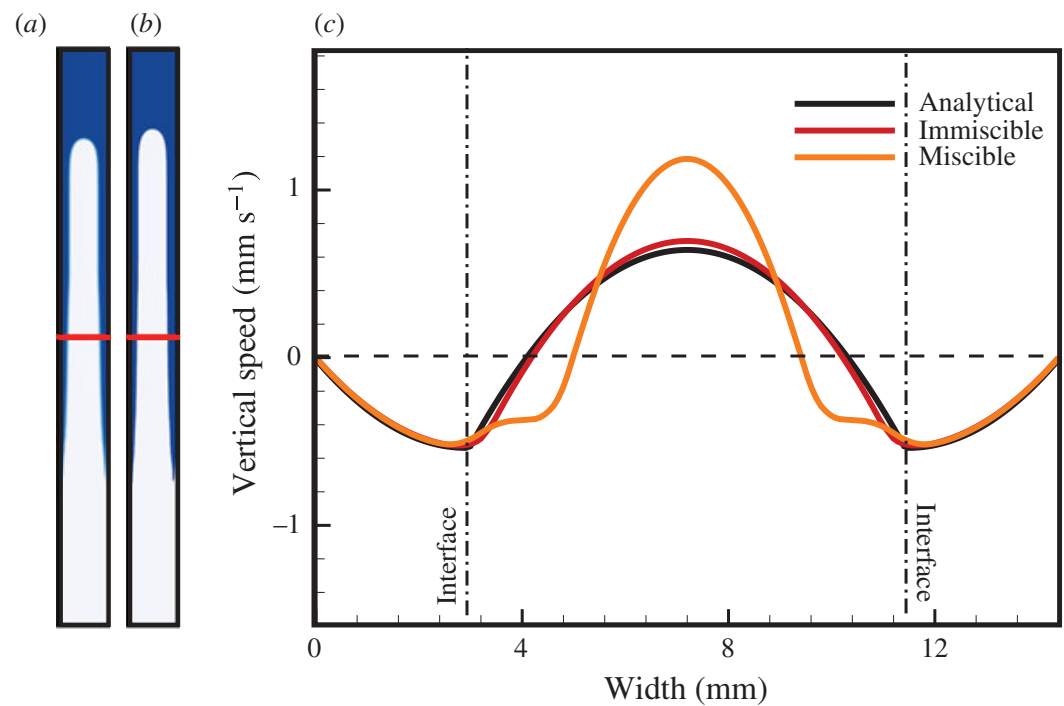

FIGURE 5. Reproduction of experiment No. 8, treating the fluids as miscible $(a)$ and immiscible $(b)$. Numerical speed profiles $(c)$ taken on marked cross-sections highlighted as red bars in $(a)$ and $(b)$ for miscible (yellow line) and immiscible (red line) flow compared to the analytical solution (black line).

the vertical flow speed across the numerical domain in an area of fully developed core-annular flow. At a given flux, the analytical model computes $\delta$ and the vertical speed curves for both solutions, but it does not allow constraining the flux from first principles. We thus use the flux computed from the numerical simulations as an input to our analytical model. The flow profile of the immiscible simulation agrees remarkably well with the analytical solution corresponding to relatively slow flow in a thick core (figure $5 c$ ) despite the fact that the analytical solution represents steady state whereas the numerical simulation is transient.

The vertical speed profile, however, is significantly modified by fluid miscibility (figure 5c). The two reasons for this modification are a gradual change in the density and viscosity and a more distributed shear stress in the interfacial zone. Despite the small amount of mixing here, the dynamic consequences are notable in both the increased maximum rise speed in the core and in the narrower upwelling portion of the flow. We find that an exponential variation of viscosity with composition magnifies this effect (see the supplementary material).

\subsection{Analysis of virtual experiments}

One of the main findings in Stevenson \& Blake (1998) is that the Poiseuille number in their experiments is essentially constant (i.e. $P s \approx 0.065$ ) at finite viscosity contrast (approximately $M>10$ ). This behaviour stands in stark contrast to the theory of Kazahaya et al. (1994), which predicts a monotonic increase of $P s$ with $M$. Figure 6 compares the range of experimentally determined $P s$ values with the numerical values resulting from our simulations and with the theoretical values calculated from our analytical model in 2-D and 3-D. We also show the theoretical values of Kazahaya et al. (1994) for comparison. Because our analytical model does not allow us to quantify the rise speed of a transient interface, we instead compute $P s$ from the 


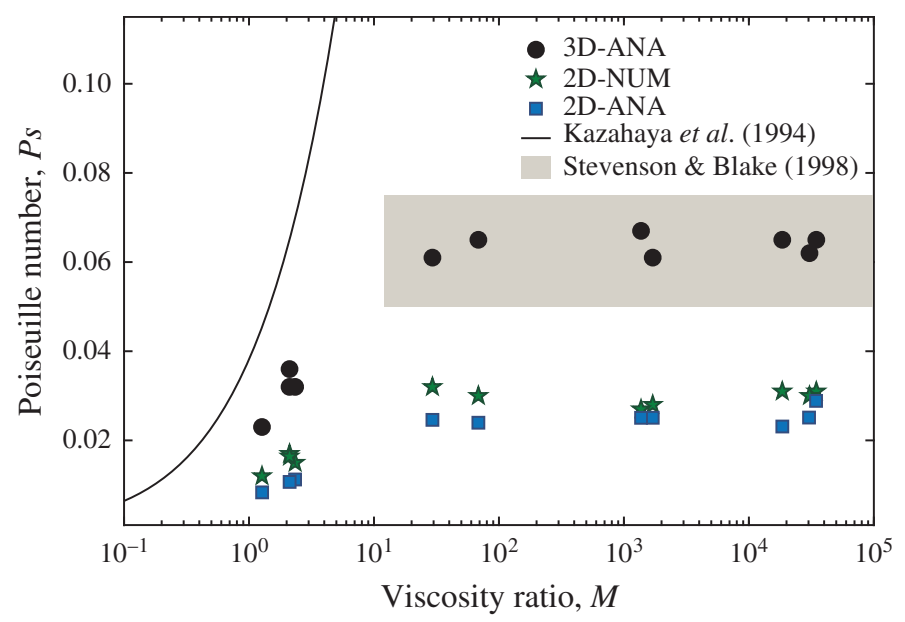

Figure 6. Plot of the Poiseuille number, $P s$, against the decadal logarithm of viscosity ratio $M$, for numerical simulations (green stars), analytical model predictions (black circles for 3-D, blue squares for 2-D), and the range of analogue experiments (grey shading) performed by Stevenson \& Blake (1998). Analytical solution of Kazahaya et al. (1994) shown for comparison (black line). The numerical simulations shown are the reproduction of the experiments of Stevenson \& Blake (1998), also shown in figure 3, and are all miscible.

average vertical flow speed in the core phase. For consistency, we use the same measure to calculate $P S$ from the numerical results. The supplementary material provides a comparison of these two metrics for rise speed and shows that they produce comparable values in our simulations.

Figure 6 shows that our 3-D analytical model (black dots) agrees well with the range of observed $P s$ numbers (grey shaded) reported by Stevenson \& Blake (1998) for $M>10$. It also quantifies the difference between $P s$ in 2-D (blue squares) versus 3-D (black circles). In 3-D, the rise speed is approximately twice as fast as in 2-D. The 2-D analytical estimates (blue squares) agree well with the 2-D numerical estimates (green stars). All three pieces, the measured data, the analytical values and the numerical outcomes, indicate that $P s$ levels off with increasing $M$, in contrast to the findings of Kazahaya et al. (1994).

Stevenson \& Blake (1998) hypothesized that the disconnection between observed Ps values and model predictions of Kazahaya et al. (1994) is related to their assumption that the interface between the ascending and descending fluid is immobile (i.e. $u_{i}=0$ ). Our virtual reproductions of the analogue experiments allow us to test this hypothesis. In figure 7, we plot vertical flow speed profiles across four different experiments: No. 10 (figure $7 a$ ), No. 9 (figure $7 b$ ), No. 5 (figure $7 c$ ) and No. 8 (figure $7 d$ ). These four experiments with increasing viscosity ratios represent the three different flow regimes indicated in figure 4. We arrange the experiments in this order to highlight the change in interface speed with increasing viscosity contrast. Our results give clear confirmation of the hypothesis of Stevenson \& Blake (1998), showing that the interface is indeed not stationary.

A finite interface speed implies that the turning point between upward- and downward-oriented flow shifts into one of the two fluids. At low viscosity contrast (flow regime III), this shift may occur in either the ascending or in the descending 
$(a 1)$
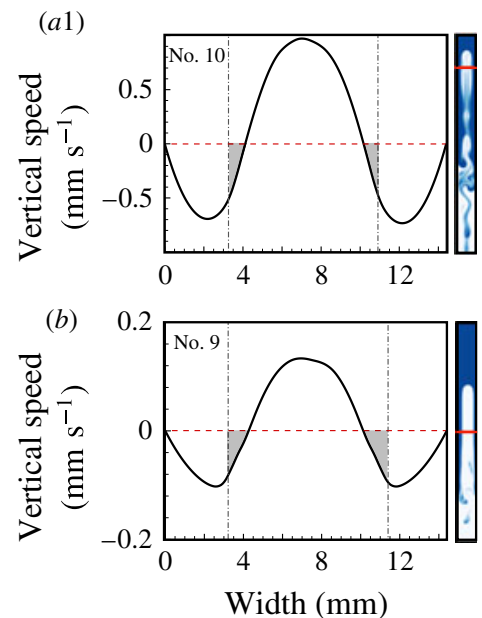

(a2)

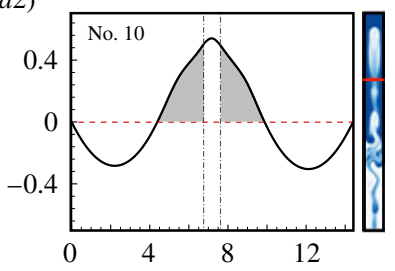

(c)

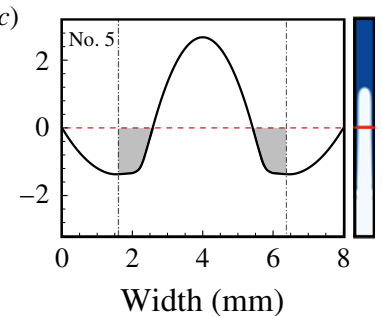

Flow-reversal zones

- Position of cross-section

Interface position, $c=0.5$

- - Flow turning point, $u=0$

FIGURE 7. Zones of flow reversal (grey shaded) in bidirectional flow experiments on horizontal profiles of vertical speed, $u(r)$, along cross-sections through virtual experiments marked by red bars across reported flow patterns. $(a 1, a 2)$ Profiles of experiment No. 10, low viscosity contrast $M$, flow regime III. (b) Experiment No. 9, intermediate $M$, regime II. (c) Experiment No. 5, high $M$, regime I. (d) Experiment No. 8, very high $M$, regime I.

fluid (see figure $7 a 1, a 2$ ) and is linked to transient effects. For flow regimes I and II, the shift occurs in the ascending fluid in all simulations (figure $7 b-d$ ). This finding implies that, at intermediate to high viscosity contrasts (i.e. $M>10$ ), a portion of the buoyant fluid in fact flows downwards in the tube - a phenomenon commonly referred to as flow reversal or backflow.

To quantify flow reversal more systematically, we compute the ratio between the flux in a given phase, $T e$, and the flow-reversal flux in the same phase, $T e_{r e v}$, which we define as

$$
T e_{\text {rev }}=\left|\int_{\delta_{\text {rev }}}^{\delta} 2 \pi r u_{a}(r) \mathrm{d} r\right|,
$$

where $\delta_{\text {rev }}$ is the point at which the vertical flow speed in the ascending fluid crosses zero, $u_{a}\left(\delta_{\text {rev }}\right)=0$. With this definition, $T e_{\text {rev }}$ quantifies the flux of ascending fluid that is trapped in the flow-reversal zone. Figure 8 shows the analytically predicted flow-reversal flux as a function of dimensionless model parameters. Figure 8(a) shows its dependence on the core radius, $\delta$, for different viscosity contrasts. For viscosity contrasts $M>10$, the experimentally observed core radii $(\delta \approx 0.6)$ cluster just below the values resulting in maximum flow reversal. In figure $8(b)$, we plot the ratio of flow reversal to total flux, again as a function of $\delta$ and $M$.

We find that at intermediate and high viscosity contrast, $T e_{\text {rev }} / T e$ is mostly insensitive to the viscosity ratio for sufficiently high core radius. In fact, for $M \gtrsim 10$ and $\delta \gtrsim 0.4$, the flow reversal takes place in the ascending phase, and both the total and flow-reversal flux approach the asymptotic limit of $M \rightarrow \infty$ :

$$
\begin{gathered}
T e(\delta, \alpha=0, M \rightarrow \infty)=\frac{\pi}{8}\left[1-4 \delta^{2}+(3-4 \log \delta) \delta^{4}\right], \\
T e_{r e v}(\delta, \alpha=0, M \rightarrow \infty)=\frac{\pi}{8} \frac{\delta^{4}\left(2 \delta^{2} \log \delta-\delta^{2}+1\right)^{2}}{\left(\delta^{2}-1\right)^{2}} \text { for } \delta>\delta_{r e v} .
\end{gathered}
$$



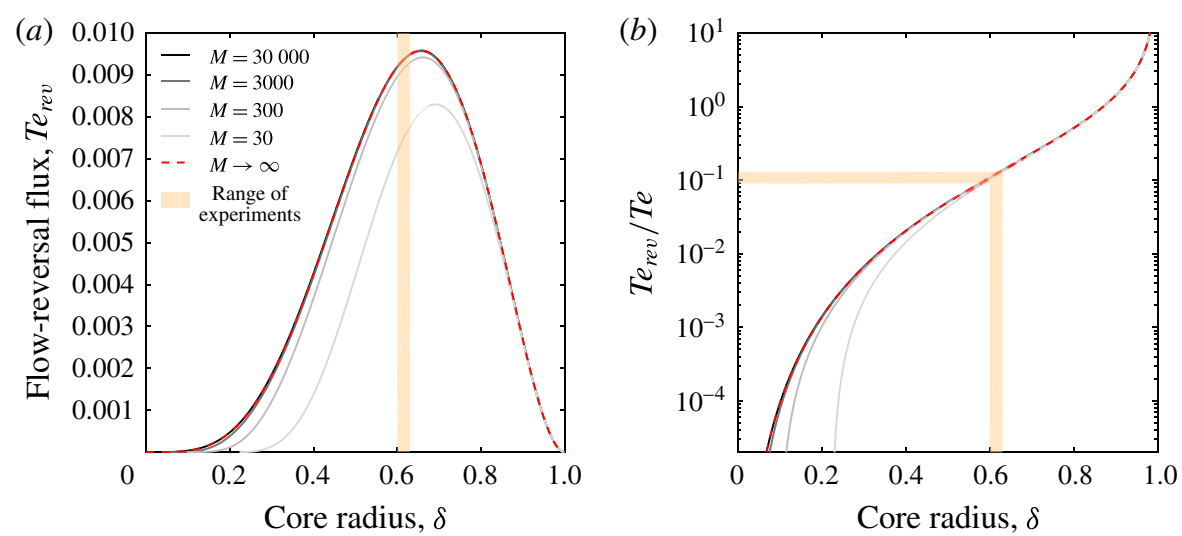

FIgURE 8. (a) Dimensionless flow-reversal flux in the ascending phase, $T e_{\text {rev }}$, as a function of the core radius $\delta$. (b) Normalized dimensionless flow-reversal flux in the ascending phase, $T e_{r e v}$, scaled by the net dimensionless flux, $T e$, as a function of the core radius, $\delta$.

The insight that the interface between the two fluids is inherently dynamic does not yet explain why $P s$ remains approximately constant with increasing $M$. To find an explanation, we return to (2.14) in $\S 2.1$. It states that the function $T e(\delta)$ has a quadratic component, which suggests that two valid solutions for $\delta$ may exist for a given flux. The existence of multiple solutions is a common feature of gravity dominated multiphase flows, yet it does not necessarily imply that both solutions are realized in the laboratory (e.g. Landman 1991; Brauner 1998; Ullmann et al. 2003; Picchi \& Poesio 2016), or indeed in natural systems.

In figure 9, we illustrate the two valid core-annular solutions for experiment No. 5 (see table 1). At the experimentally observed dimensionless flux, $T e=0.075$, our model predicts two solutions for the core radius, the thick-core ( $\delta_{\text {thick}}$, blue diamond) and the thin-core solution ( $\delta_{\text {thin }}$, red triangle). The two corresponding flow profiles, shown from the centre of the tube to the wall in figure $9(b, c)$, highlight that the same overall flux can be accommodated by either a thin, rapidly ascending core with a thick annulus, or through a thick, slowly ascending core with a thin annulus. Interestingly, both solutions are far removed from the point of maximum flux or flooding point (yellow $\times$ ).

Table 1 lists the two analytically computed core radii at the $T e$ values inferred from the reported terminal rise speeds in all 11 experiments. The solutions compatible with Stevenson \& Blake's (1998) experimental and our numerical outcomes are printed in bold. We find that all experiments with viscosity contrasts of $M>10$ select the thick-core solution, $\delta_{\text {thick}}$. The thin-core solution, $\delta_{\text {thin }}$, may be pertinent for the experiments with small viscosity contrasts (experiments No. 3, No. 4, No. 10 and No. 11 ), but these cases do not exhibit stable core-annular flow, and the applicability of the analytical model is thus questionable.

Figure 10 shows analytical flux solutions for all experiments as a function of core radius and viscosity contrast. For each $T e-\delta$ curve in figure $10(a)$, we mark the solution that is realized in experiments $\left(\delta_{\text {thick }}\right.$, red triangle; $\delta_{\text {thin }}$, blue diamond). We show curves for experiments No. 3, No. 4, No. 10 and No. 11 as light-grey lines to convey that our analytical model is questionable for these cases. The experiments with $M>3$ (solid lines) all cluster around $\delta \approx 0.61$ and $T e \approx 0.075$. Hence, the scatter in the $P s$ number 

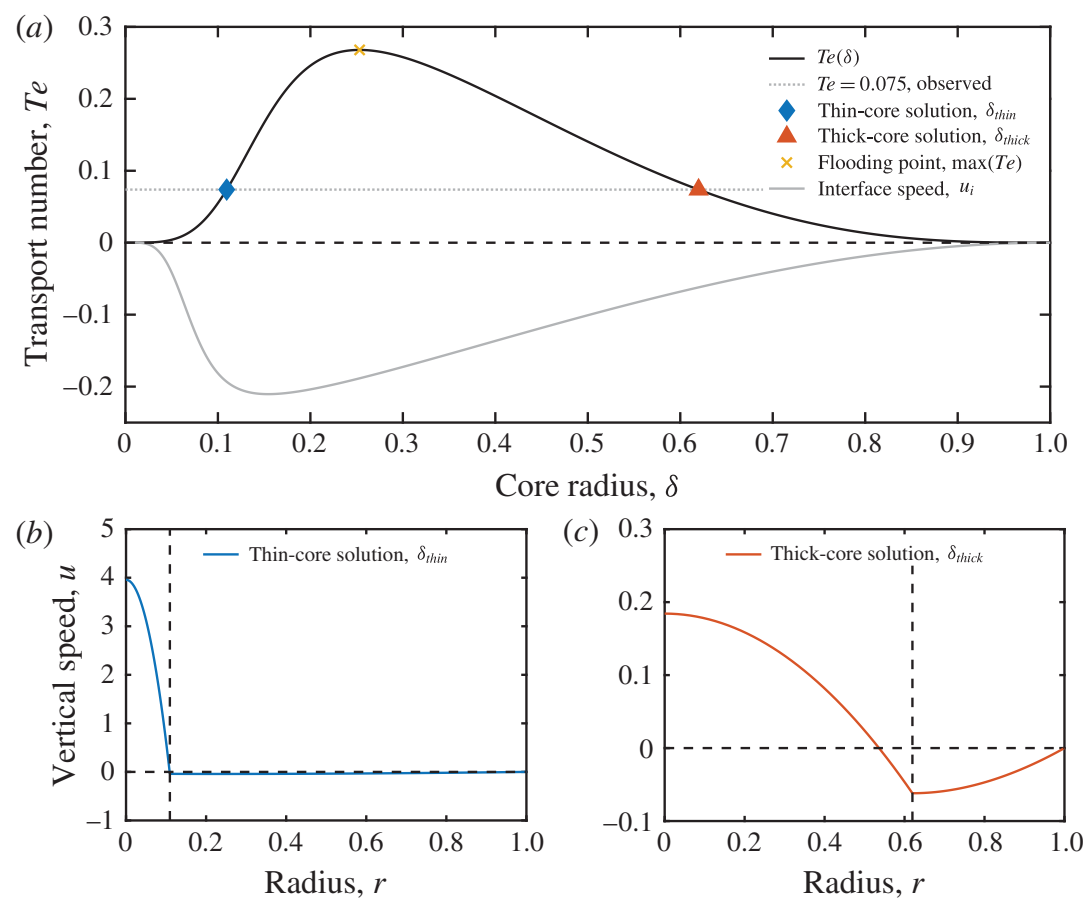

FIGURE 9. (a) Transport number, Te, and interface speed, $u_{i}$, as a function of the dimensionless core radius, $\delta$, for experiment No. $5(M=1700)$. $(b, c)$ Dimensionless velocity profiles of the thin-core, $\delta_{\text {thin }}$, and thick-core, $\delta_{\text {thick}}$, solutions at $T e=0.075$. We estimate $T e$ based on the experimental rise speed.

in Stevenson \& Blake (1998) (see figure 6) is not related entirely to uncertainty of measurement but also reflects the fact that $T e$ values at different $M>3$ are very similar but not identical (see figure $10 b$ ). For experiments with a viscosity ratio close to unity (light-grey lines), Te assumes much lower values (figure 10b), and the two possible solutions are no longer clearly distinct.

This analysis suggests that the dichotomy in Ps number observed by Stevenson \& Blake (1998) and reproduced in figure 6 reflects a shift from thick-core core-annular flow (flow regimes I and II) to unstable overturn flow (flow regime III). Contrary to the thin-core solution, the thick-core solution exhibits approximately constant core thickness over a large range of $M$ (see figure $10 a$ ), which means that a constant $T e$ translates to a constant Ps (2.12). If any of the experiments exhibited the thin-core solution, $P s$ would increase with $M$.

\subsection{Simulations of persistent exchange flow}

The result that only the thick-core solution is realized in core-annular flow at finite viscosity contrast in closed tubes raises the question whether this flow configuration is generally preferable. At very high viscosity contrast, $M>10000$, the thin-core solution entails an extremely thin core (e.g. see experiments No. 2, No. 7 and No. 8 in table 1), which would be highly prone to wave bridging and flow collapse (e.g. Barnea 1987). In the limit of extreme to infinite viscosity contrast, it is hence likely that the thickcore solution is generally preferable. 

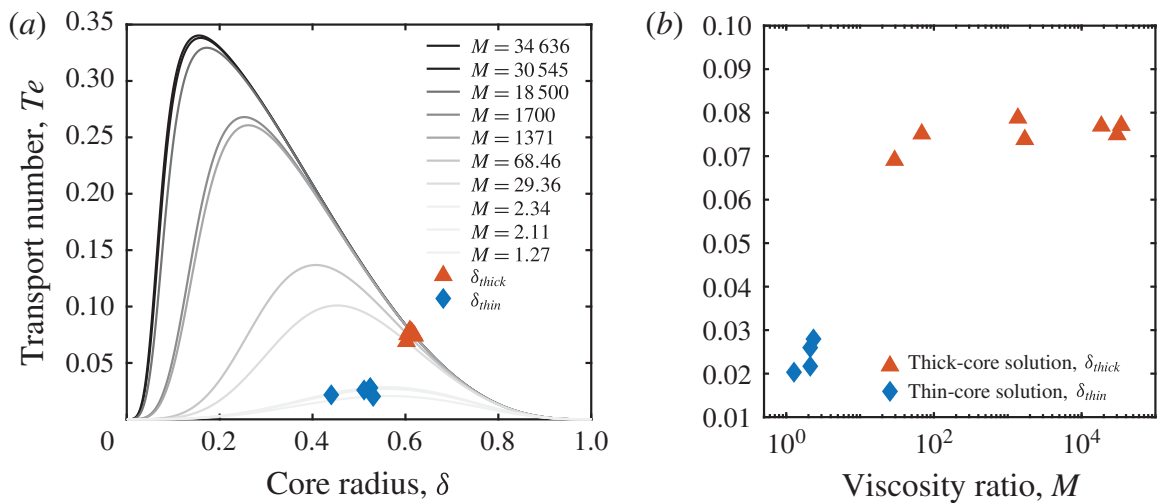

Figure 10. (a) $T e-\delta$ curves for all experiments listed in table 1 . The plot shows 10 instead of 11 curves, because experiments No. 10 and No. 11 have the same viscosity ratio. Numerically and experimentally realized core radii are highlighted as red triangles (thick core) and blue diamonds (thin core). (b) Transport number, Te, against the viscosity ratio, $M$, as computed from the analytical model for the experiments of Stevenson \& Blake (1998).

Determining the physical relevance of the two solutions based on core radius alone, however, becomes unsatisfactory at high to intermediate viscosity contrast, when the core radii become increasingly comparable. In this section, we explore the possibility that the physically pertinent solution may be controlled not only by the material parameters but also by the in- and outflux boundaries, an idea raised but not explored in detail by Barnea \& Taitel (1985). The experiments of Stevenson \& Blake (1998) were performed in closed test tubes, which are significantly different from natural systems where exchange flow is typically the consequence of continued flux.

To generalize insights from closed to open systems, we perform simulations with open boundaries at the top and the base of the model domain. All the simulations discussed in this section are forced by a time-independent inflow condition imposed along the base of the domain and set according to the analytical speed profile in 2-D (see (8) and (9) in the supplementary material). This choice of boundary condition automatically imposes a certain flux, Te. We test both thin- and thick-core influx by applying either $\left.u\right|_{\text {base }}(r)=u_{\text {thin }}(r)$ or $\left.u\right|_{\text {base }}(r)=u_{\text {thick }}(r)$. Both solutions entail the same flux, Te. We continue to enforce a no-slip condition $\left(\left.\boldsymbol{v}\right|_{\text {side }}=0\right)$ along the side walls. For the outflow condition and the initial interface position, we consider four different cases (see figure 11).

For the first case (figure $11 a 1-c$ ), we impose a fixed outflow condition along the top boundary. We set the same analytical flow profile for the top and the base of the domain, that is $\left.u\right|_{\text {base }}(r)=\left.u\right|_{\text {top }}(r)=u_{\text {thick }}(r)$ and $\left.u\right|_{\text {base }}(r)=\left.u\right|_{\text {top }}(r)=u_{\text {thin }}(r)$, respectively. We also initiate the concentration field to the corresponding geometry extended through the whole domain (see figure 11a1,b1). This choice implies that the interface is pinned at both the top and bottom of the domain. Although this set-up is clearly contrived, it is an interesting end-member case for understanding the respective stability of the two solutions. Intuitively, one might expect that, if forced by the analytical solution on both ends, the flow field in the domain will remain close to that solution. Figure 11(c) shows that this is clearly not the case. Although both interfaces are slightly wavy initially (figure $11 a 1, b 1$ ), the thick-core solution stabilizes (figure 11a3). However, the thin-core case begins transitioning to the thick-core flow 
Thick film

Thin film
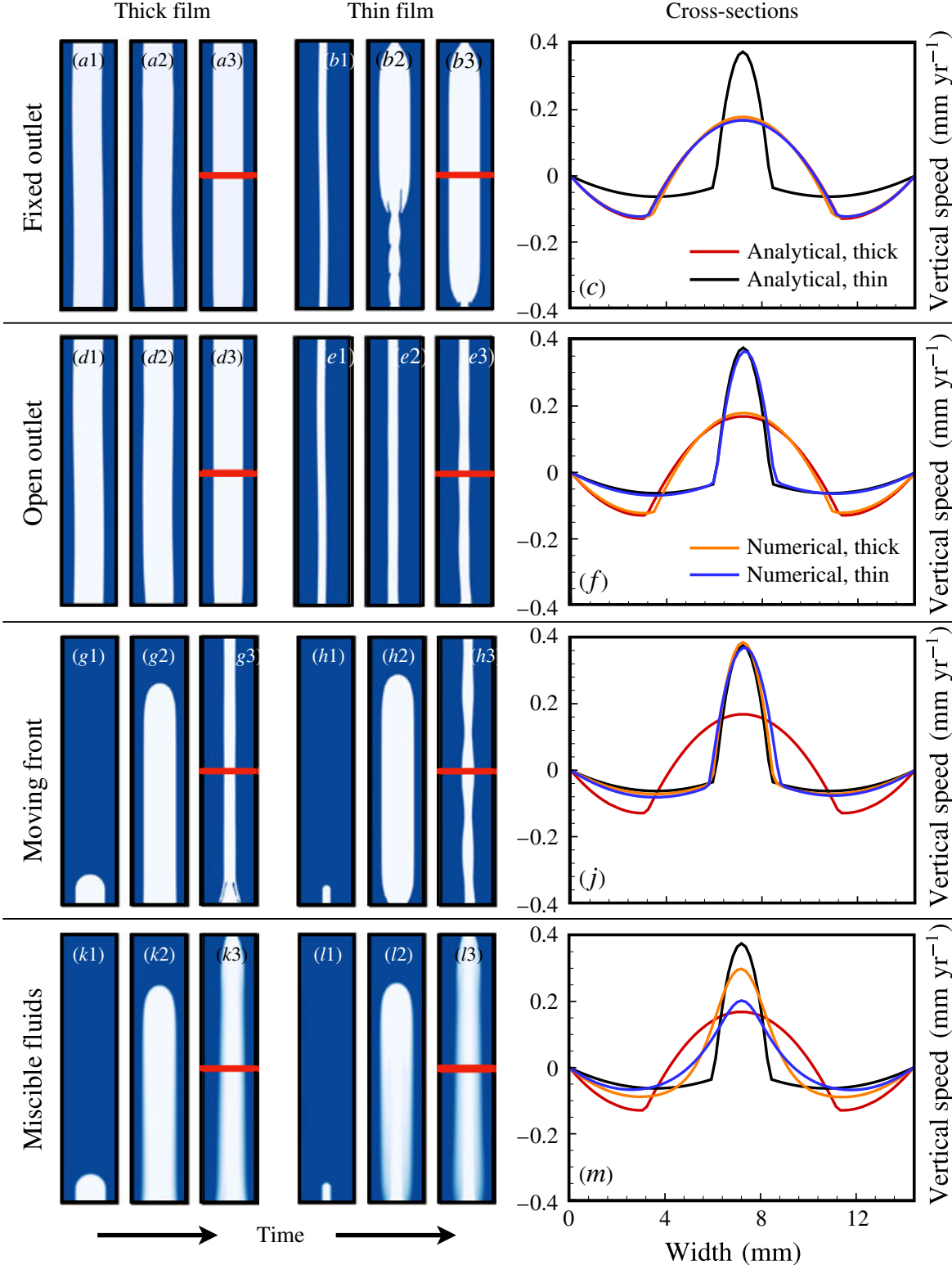

FIGURE 11. Snapshots of numerical simulations forced with thick- and thin-core analytical solutions at the inlet. Material properties are identical to those in experiment No. 9 of Stevenson \& Blake (1998). Results are shown for: fixed outlet boundary, enforcing the analytical model at the top boundary for thick- $(a 1-a 3)$ and thin-core (b1-b3) solutions; free outlet, stress-free top boundary allowing free flow through the top, starting from fully developed bidirectional configuration $(d 1-e 3)$, and with a transient front moving through the domain $(g 1-h 3)$; the same as in the previous case but with miscible fluids $(k 1-l 3)$. Horizontal profiles of vertical speed $(c, f, j, m)$ show numerical solutions approaching either thin- or thick-core analytical solutions depending on boundary conditions and fluid miscibility. See also supplementary movie 4 for a simulation of $(h 1-h 3)$. 
field from the top boundary downwards almost immediately after the onset of flow (figure 11b2,b3). The speed profiles taken in the middle of the domain, where the flow is approximately at steady state, confirm that both simulations closely approach the analytical thick-core solution (figure 11c).

The second case (figure 11d1-f) represents a scenario with open outflow across a stress-free top boundary, which we enforce by setting $\left.p\right|_{\text {top }}=$ const., and $\partial \boldsymbol{v} /\left.\partial z\right|_{\text {top }}=\mathbf{0}$. The flow field across the upper boundary thus evolves over time, and the interface will move freely with the outflow. The initial position of the interface is identical to that in the first case (figure 11d1,e1). The emerging flow fields correspond closely to the analytical solution imposed at the base of the domain. A thick-core inflow leads to a stable thick-core configuration (figure 11d3) and a thin-core inflow leads to a stable thin-core configuration (figure 11e3). This case demonstrates that the thin-core solution is a physically relevant flow configuration, at least at a viscosity contrast of $M \approx 30$. Together with the first case, these simulations confirm that boundary conditions indeed have a profound influence on which mathematically valid flow configuration is realized in practice, as suggested by Barnea \& Taitel (1985).

One advantage of the variable over the fixed outflow case is that we can now consider a different initial interface position. In our third case (figure 11g1-j), we initiate a closed interface confined to the vicinity of the lower boundary (figure $11 g 1, h 1$ ), ensuring that the core radius corresponds to the analytical solution enforced at inflow. In these simulations, the interface movement through the domain is similar to that in the experiments (figure $11 g 2, h 2$ ), but the interface eventually leaves the computational domain through the upper boundary.

Immediately after onset, both the thick- and thin-core flows approach the thick-core solution (figure 11g2,h2). However, as soon as the interface intersects with the upper boundary, the flow in both simulations collapses back to the thin-core solution (figure $10 g 3, h 3$ ). This case demonstrates not only that the preferable flow configuration depends on the boundary conditions, but also that a transient disruption on one boundary, such as the passage of the interface through the top, can trigger a switch in the flow configuration that propagates across the entire domain.

Because the analytical solution strictly applies only to the immiscible limit, we have thus far considered only immiscible fluids in this section. In the fourth case (figure $11 k 1-m$ ), we repeat the third case with miscible fluids at high $\mathrm{Pe}$. Initially, the thin-core flow widens immediately (figure 11l2), but both flow configurations collapse back to a thin core once the interface passes through the upper boundary (figure $11 k 3, l 3$ ). The vertical speed profiles are reminiscent of the thin-core solution but more spread out (figure $11 \mathrm{~m}$ ). The interface remains less wavy throughout, which supports the theoretical expectation that mixing stabilizes core-annular flow against interface waves (e.g. Meiburg et al. 2004).

\section{Discussion}

\subsection{Theoretical implications}

Laboratory experiments have provided invaluable insights into the dynamics of buoyancy-driven exchange flows, but they inevitably simplify the more complex flow problem they intend to represent. One aspect in which many analogue models of exchange flow differ fundamentally from natural or industrial systems is that bidirectional flow occurs only as transient behaviour until a steady state of complete inversion of the two fluids is reached (Stevenson \& Blake 1998; Huppert \& Hallworth 2007; Beckett et al. 2011; Palma, Blake \& Calder 2011). The steady state in the 
laboratory is hence very different from that in most natural or industrial problems of interest. Our analysis suggests that this difference may be consequential, because closed systems realize only a subset of the possible flow solutions that occur in open systems. Experiments in closed domains may hence systematically underestimate the dynamic variability of open-system flow.

We derive a simple analytical model that allows us to characterize the two steadystate solutions for core-annular flow. These two solutions refer to the same magnitude of flux, or $T e$, but differ in their core radii, $\delta_{\text {thick }}$ and $\delta_{\text {thin }}$, flow speed profile, and degree of flow reversal. We also predict the flux curve, $T e(\delta)$, based only on the fluid properties and tube radius. The model does not require a fitting parameter to match experimental or numerical data, but it predicts neither the total flux nor the solution that is realized to achieve that flux. In fact, our analysis implies that it is not possible to predict these two outcome variables based on the fluid properties and the tube geometry alone, because the boundary conditions play an important role in determining these variables.

Huppert \& Hallworth (2007) suggested that buoyancy-driven exchange flow in vertical pipes tends to maximize flux, which would be equivalent to maximizing $T e$. This argument implies that the flux should be at the flooding point for most or all of the experiments (figure 10a), because the non-dimensional viscous dissipation is directly proportional to the flux magnitude $T e$ (see the supplementary material). This prediction, however, is at odds with both the experimental and the numerical results discussed here. Our results confirm prior conjectures by Joseph et al. (1984) and Beckett et al. (2011) that the viscous dissipation principle does not generally hold in bidirectional flow.

Thus far, our finding that the boundaries and flow history select the realized solution has been based only on direct numerical simulations in 2-D. Although the flow regimes observed in these simulations agree both qualitatively and quantitatively with laboratory data, interface instabilities scale differently in 2-D than in 3-D, and extrapolating from a 2-D numerical simulation to a 3-D natural system is not trivial.

To ensure the robustness of our results, we reanalyse the laboratory experiments by Beckett et al. (2011) to verify that both solutions are observable at high viscosity contrast in open systems. Their laboratory set-up mimics an open-system, buoyancy-driven exchange flow by connecting a vertical tube to fluid reservoirs at both ends (e.g. Huppert \& Hallworth 2007; Beckett et al. 2011; Palma et al. 2011). The flow patterns that arise in this more complex geometry are more varied than those in Stevenson \& Blake (1998). By reanalysing the measured velocity profiles from Beckett et al. (2011), we find that their experiments No. $20(M \approx 1175)$, No. 15 $(M \approx 377)$ and No. $11(M \approx 144)$ correspond to the thin-core solution, that experiment No. 9 is close to maximum flux where only one solution exists, and that experiments No. 17 and No. 16 realize the thick-core solution. Our analysis of their data is shown in figure 12, where we give the $T e(\delta)$ curves and identify realized solutions for all experiments in their figure 9 (figure 12a). The experimental data of Beckett et al. (2011) hence support our numerically derived insight that both solutions are pertinent for understanding exchange flow in open systems at viscosity contrasts up to three orders of magnitude.

\subsection{Ramifications for volcanic systems}

In its current form, our model is not yet suitable for a direct quantitative comparison with field data from a specific volcano. Some of our insights, however, may inform 

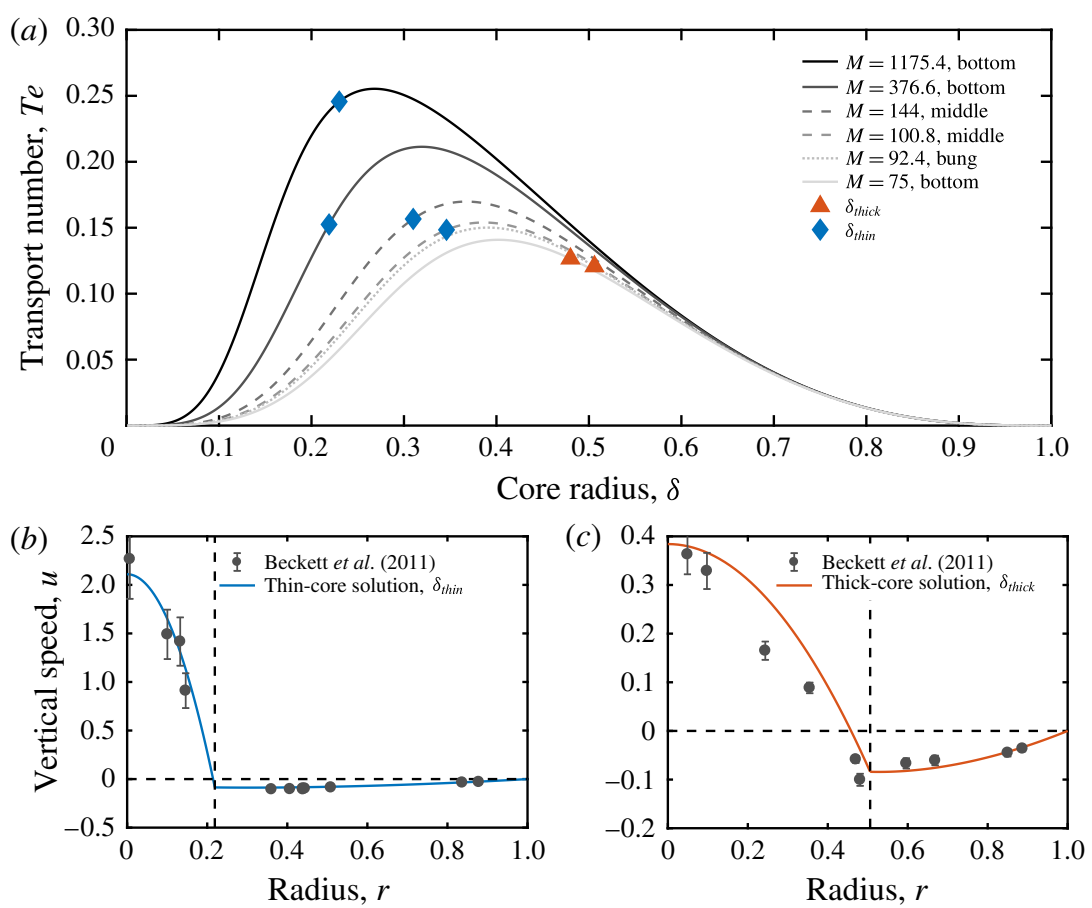

FIGURE 12. Reappraisal of the laboratory experiments performed by Beckett et al. (2011). (a) $T e-\delta$ curves and realized solutions for experiments No. 9, No. 11, No. 15, No. 16, No. 17 and No. 20 based on material parameters provided in Beckett et al. (2011). (b) Experiment No. 15 exhibits the thin-core solution despite a significant viscosity contrast of $M \approx 377$. (c) Experiment No. 17 exhibits the thick-core solution at $M \approx 92$, demonstrating that the viscosity contrast is not the main factor determining the respective stability of the two core-annular solutions at intermediate to high viscosity ratio.

the interpretation of field data on a conceptual level. One pertinent insight is that a change at either the base of a conduit or its opening in a volcanic crater could trigger a switch in the flow regime in the conduit. The effect of different flow regimes in the conduit on eruptive surface processes was well explored and reviewed by Vergniolle \& Mangan (2000). Our results suggest that the reverse is possible as well: eruptive surface processes can alter the flow regime in the volcanic conduit. For example, a disruption at the free surface, which might arise during an eruption or other events such as mass movements in the crater, could trigger a switch in the flow configuration that is realized in the conduit. Of course, our simplistic boundary conditions (figure $11 d 1-e 3$ and $g 1-h 3$ ) do not adequately represent eruptive processes at a free surface. Nonetheless, our results demonstrate the potentially significant role of surface conditions in selecting a flow regime in the conduit.

We argue that the existence of two different, stable flow configurations could be reflected in erupted field samples from persistently degassing volcanoes, potentially from different stages of the same eruption. A switch from thick- to thin-core flow could increase the magma ascent rate by more than an order of magnitude, which may be detectable in microanalytical data. Our results show that if a change in the estimated or measured ascent speed of the magma is detected, this observation does 

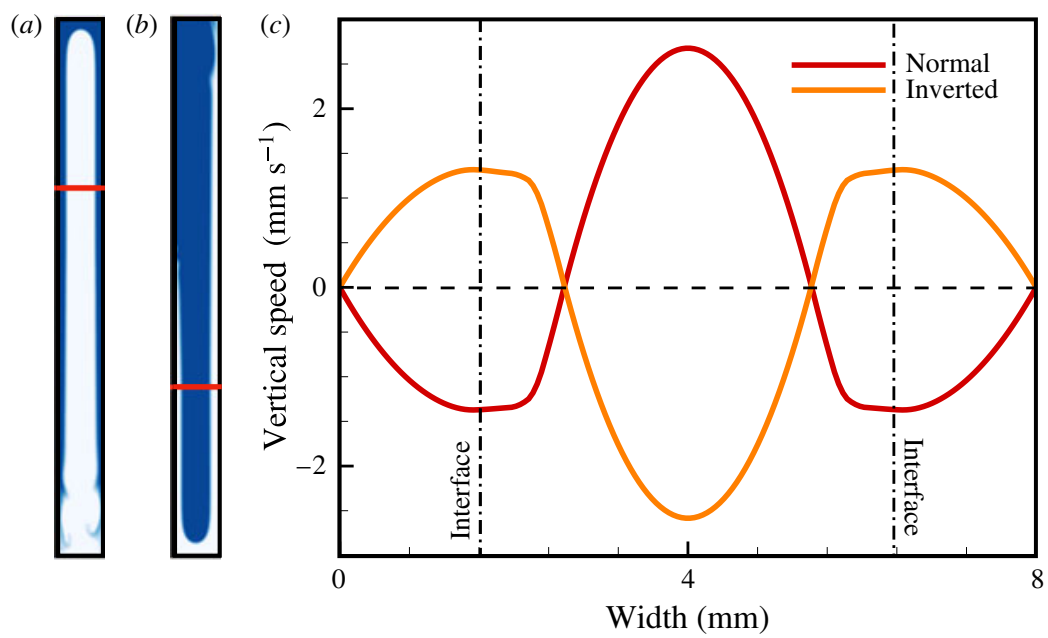

FIGURE 13. Comparison between the reproduction of experiment No. $5(a)$ and an additional simulation with inverted viscosity contrast $1 / M(b)$, where the heavy and now less viscous fluid (dark blue) forms a sinking core. $(c)$ Horizontal speed profiles for the inverted (yellow line) and normal (red line) viscosity contrast case.

not necessarily require additional magma supply or a change in the volatile influx at depth. It could also be related to flow switching in the volcanic conduit.

Another potentially relevant insight of our study is the significant flow reversal present in all three of the flow regimes identified by Stevenson \& Blake (1998). This result suggests that flow reversal might be the norm rather than the exception in volcanic conduits. Our analytical model predicts that flow reversal occurs in the less viscous phase, a finding that is corroborated by our simulations (see figure 13 and additional results in the supplementary material). Typically, the buoyant, volatile-rich magma has the lower viscosity, as was assumed in the experiment of Stevenson \& Blake (1998). In that case, flow-reversal flux is oriented downward, which raises the question of whether the magma trapped in flow reversal is simply cycled back into a crustal reservoir never to be sampled by eruption, or whether some magma may circulate in the conduit for some time before being erupted. In the latter scenario, continued cycling along with mixing between different magma batches may lead to fundamentally different compositional evolution of both the magma and its volatile load than would be expected from a straight decompression path.

Flow reversal may also increase mixing between volatile-poor and volatile-rich magmatic melt, because the two fluids move in the same direction in some portion of the conduit. As demonstrated in figure $5(b, c)$, even a small amount of mixing could have important dynamic ramifications for conduit flow. As pointed out by Witham (2011), magma mixing during conduit convection could be relevant for understanding why observed melt-inclusion trends from persistently degassing volcanoes rarely coincide with modelled degassing trends, as recently reviewed in Métrich \& Wallace (2008).

\section{Conclusions}

In this study, we reproduce, explain, and generalize laboratory experiments of buoyancy-driven exchange flow in vertical tubes. We derive an analytical model for 
core-annular flow - the most commonly observed configuration of bidirectional flow - that is consistent with laboratory observations and direct numerical simulations. The primary objective of this paper is to understand the variability of core-annular flow in the laboratory context, but our model may also provide a suitable starting point for integrating the additional complexity needed for quantifying conduit flow in persistently degassing volcanoes. The key finding of our analysis is that core-annular flow is bistable at finite viscosity contrast and that the pressure and fluxes at the boundaries of the domain, along with the transient history of the flow, play an important role in selecting the realized flow solution. This result implies that buoyancy-driven exchange flow is not uniquely determined by the material properties of the fluids and geometric parameters of the tube.

\section{Author contributions}

J.S. conceptualized the study, integrated the results from the numerical and the analytical model and wrote the paper. Z.Q. performed the numerical simulations and compiled the supplementary material. D.P. performed the analytical derivation and computations. T.K. assisted with the numerical modelling, creation of figures and the text. I.B. provided feedback on the study conception and the text.

\section{Acknowledgements}

The authors would like to thank F. Beckett for providing us with the laboratory data and two anonymous reviewers for helpful comments. E. Dunham and two anonymous reviewers provided insightful remarks that have helped improve this manuscript considerably. T.K. is grateful for support from the Swiss National Science Foundation's Postdoc Mobility Fellowship no. P300P2_177816. The source code used in this study is available through GitLab at http://zapad.stanford.edu/sigma/JFM2018-bidirectional.

\section{Supplementary material and movies}

Supplementary material and movies are available at https://doi.org/10.1017/jfm. 2018.382

\section{REFERENCES}

Arakeri, J. H., Avila, F. E., Dada, J. M. \& Tovar, R. O. 2000 Convection in a long vertical tube due to unstable stratification - A new type of turbulent flow? Curr. Sci. 79, 859-866.

BAI, R., Chen, K. \& Joseph, D. D. 1992 Lubricated pipelining: stability of core-annular flow. Part 5. Experiments and comparison with theory. J. Fluid Mech. 240, 97-132.

BARNEA, D. 1987 A unified model for predicting flow-pattern transitions for the whole range of pipe inclinations. Intl J. Multiphase Flow 13, 1-12.

Barnea, D. \& TAitel, Y. 1985 Stability of annular flow. Intl Commun. Heat Mass Transfer 12, 611-621.

Beckett, F. M., Mader, H. M., Phillips, J. C., Rust, A. C. \& Witham, F. 2011 An experimental study of low-Reynolds-number exchange flow of two Newtonian fluids in a vertical pipe. J. Fluid Mech. 682, 652-670.

BRAUner, N. 1998 Liquid-liquid two-phase flow. In Modelling and Experimentation in Two-phase Flow, pp. 221-279. Springer.

Burton, M. R., Mader, H. M. \& Polacci, M. 2007 The role of gas percolation in quiescent degassing of persistently active basaltic volcanoes. Earth Planet. Sci. Lett. 264 (1), 46-60. 
Chen, K., BAI, R. \& Joseph, D. D. 1990 Lubricated pipelining. Part 3. Stability of core-annular flow in vertical pipes. J. Fluid Mech. 214, 251-286.

Chen, K. \& Joseph, D. D. 1991 Lubricated pipelining: stability of core-annular flow. Part 4. Ginzburg-Landau equations. J. Fluid Mech. 227, 587-615.

Dalziel, S. B. 1992 Maximal exchange in channels with nonrectangular cross sections. J. Phys. Oceanogr. 22, 1188-1206.

Francis, P., Oppenheimer, C. \& Stevenson, D. 1993 Endogenous growth of persistently active volcanoes. Nature 366, 554-557.

FrigaARD, I. A. \& SCHERzer, O. 1998 Uniaxial exchange flows of two bingham fluids in a cylindrical duct. IMA J. Appl. Maths 61, 237-266.

Goyal, N. \& Meiburg, E. 2006 Miscible displacements in Hele-Shaw cells: two-dimensional base states and their linear stability. J. Fluid Mech. 558, 329-355.

Hickox, C. E. 1971 Instability due to viscosity and density stratification in axisymmetric pipe flow. Phys. Fluids 14, 251-262.

HU, H. H. \& Joseph, D. D. 1989 Lubricated pipelining: stability of core-annular flow. Part 2. J. Fluid Mech. 205, 359-396.

Hu, H. H. \& Patankar, N. 1995 Non-axisymmetric instability of core-annular flow. J. Fluid Mech. 290, 213-224.

Huppert, H. E. \& Hallworth, M. A. 2007 Bi-directional flows in constrained systems. J. Fluid Mech. 578, 95-112.

Joseph, D. D., Bai, R., Chen, K. P. \& Renardy, Y. Y. 1997 Core-annular flows. Annu. Rev. Fluid Mech. 29, 65-90.

Joseph, D. D., Renardy, M. \& Renardy, Y. 1984 Instability of the flow of two immiscible liquids with different viscosities in a pipe. J. Fluid Mech. 141, 309-317.

Joseph, D. D. \& Renardy, Y. Y. 1992 Fundamentals of Two-fluid Dynamics. Springer.

Kazahaya, K., Shinohara, H. \& Saito, G. 1994 Excessive degassing of Izu-Oshima volcano: magma convection in a conduit. Bull. Volcanol. 56, 207-216.

LANDMAN, M. J. 1991 Non-unique holdup and pressure drop in two-phase stratified inclined pipe flow. Intl J. Multiphase Flow 17, 377-394.

McBirney, A. R. \& Murase, T. 1984 Rheological properties of magmas. Annu. Rev. Earth Planet. Sci. 12, 337-357.

Meiburg, E., Vanaparthy, S. H., Payr, M. D. \& Wilhelm, D. 2004 Density-driven instabilities of variable-viscosity miscible fluids in a capillary tube. Ann. N.Y. Acad. Sci. 1027, 383-402.

Métrich, N.\& WAllace, P. J. 2008 Volatile abundances in basaltic magmas and their degassing paths tracked by melt inclusions. Rev. Mineral. Geochem. 69, 363-402.

Palma, J. L., Blake, S. \& CAlder, E. S. 2011 Constraints on the rates of degassing and convection in basaltic open vent volcanoes. Geochem. Geophys. Geosyst. 12, Q11006.

Petitjeans, P. \& Maxworthy, T. 1996 Miscible displacements in capillary tubes. Part 1. experiments. J. Fluid Mech. 326, 37-56.

PiCCHI, D. \& Poesio, P. 2016 Stability of multiple solutions in inclined gas/shear-thinning fluid stratified pipe flow. Intl J. Multiphase Flow 84, 176-187.

Preziosi, L., Chen, K. \& Joseph, D. D. 1989 Lubricated pipelining: stability of core-annular flow. J. Fluid Mech. 201, 323-356.

Qin, Z., Delaney, K., Riaz, A. \& Balaras, E. 2015 Topology preserving advection of implicit interfaces on Cartesian grids. J. Comput. Phys. 290, 219-238.

QIN, Z.\& SuCKALE, J. 2017 Direct numerical simulations of gas-solid-liquid interactions in dilute fluids. Intl J. Multiphase Flow 96, 34-47.

Ray, E., Bunton, P. \& Pojman, J. A. 2007 Determination of the diffusion coefficient between corn syrup and distilled water using a digital camera. Am. J. Phys. 75, 903.

RENARDY, Y. Y. 1997 Snakes and corkscrews in core-annular down-flow of two fluids. J. Fluid Mech. 340, 297-317.

Russell, T. W. F. \& Charles, M. E. 1959 The effect of the less viscous liquid in the laminar flow of two immiscible liquids. Can. J. Chem. Engng 37, 18-24. 
Scoffoni, J., Lajeunesse, E. \& Homsy, G. M. 2001 Interface instabilities during displacements of two miscible fluids in a vertical pipe. Phys. Fluids 13, 553-554.

Sethian, J. A. 1996 Level Set Methods and Fast Marching Methods. Cambridge University Press.

Stevenson, D. S. \& Blake, S. 1998 Modelling the dynamics and thermodynamics of volcanic degassing. Bull. Volcanol. 60, 307-317.

Suckale, J., Hager, B. H., Elkins-Tanton, L. T. \& Nave, J.-C. $2010 a$ It takes three to tango: 2. Bubble dynamics in basaltic volcanoes and ramifications for modeling normal Strombolian activity. J. Geophys. Res. 115, B07410.

Suckale, J., Nave, J.-C. \& Hager, B. H. $2010 b$ It takes three to tango: 1. Simulating buoyancydriven flow in the presence of large viscosity contrasts. J. Geophys. Res. 115, B07409.

Suckale, J., Sethian, J. A., Yu, J. \& Elkins-Tanton, L. T. 2012 Crystals stirred up: 1. Direct numerical simulations of crystal settling in nondilute magmatic suspensions. J. Geophys. Res. 117, E08004.

TAN, C. T. \& Homsy, G. M. 1986 Stability of miscible displacements in porous media: rectilinear flow. Phys. Fluids 29, 3549-3556.

Ullmann, A. \& BRAUner, N. 2004 Closure relations for the shear stress in two-fluid models for core-annular flow. Multiphase Sci. Technol. 16 (4), 355-387.

Ullmann, A., Zamir, M., Ludmer, Z. \& Brauner, N. 2003 Stratified laminar countercurrent flow of two liquid phases in inclined tubes. Intl J. Multiphase Flow 29, 1583-1604.

Vanaparthy, S. H., Meiburg, E. \& Wilhelm, D. 2003 Density-driven instabilities of miscible fluids in a capillary tube: linear stability analysis. J. Fluid Mech. 497, 99-121.

Vergniolle, S. \& MAngan, M. 2000 Hawaiian and Strombolian eruptions. In Encyclopedia of Volcanoes, pp. 447-461. Elsevier.

Witham, F. 2011 Conduit convection, magma mixing, and melt inclusion trends at persistently degassing volcanoes. Earth Planet. Sci. Lett. 301, 345-352. 